\title{
Spatial and Spectral Nonlinear Beam Control with Active Multimode Graded Index Fiber Taper
}

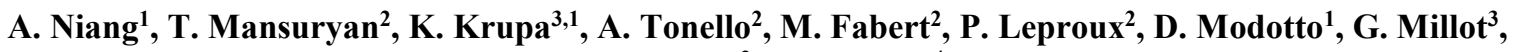 \\ V. Couderc ${ }^{2}$, S. Wabnitz ${ }^{4}$ \\ 1. Dipartimento di Ingegneria dell'Informazione, Università degli Studi di Brescia, via Branze 38, 25123, Brescia, Italy \\ 2. Université de Limoges, XLIM, UMR CNRS 7252, 123 Avenue A. Thomas, 87060 Limoges, France \\ 3. Université Bourgogne Franche-Comté, ICB, UMR CNRS 6303, 9 Avenue A. Savary, 21078 Dijon, France \\ 4. DIET, Sapienza Università di Roma, via Eudossiana 18, 00184 Rome, Italy \\ Author e-mail address: alioune.niang@unibs.it
}

\begin{abstract}
We experimentally demonstrate spatial beam self-cleaning in tapered Ytterbium-doped multimode fiber with parabolic index and doping profile in passive and active configurations. We also obtained supercontinuum emission in the range $520 \mathrm{~nm}-2600 \mathrm{~nm}$. (๑) 2019 The Author(s)
\end{abstract}

OCIS codes: (190.0190) Nonlinear optics, (060.0060) fiber optics, (190.3270) Kerr effect, (190.5940) self-action effect

Multimode optical fibers are receiving a renewed interest, as they permit the control of complex spatio-temporal nonlinear processes [1]. Among these, spatial Kerr-beam self-cleaning (KBSC) in graded index (GRIN) multimode optical fibers (MMFs) transforms an output speckled beam in a quasi-single-mode beam, such that most of the beam energy remains confined in a single, low-order transverse mode of the fiber [2]. A specific output mode can be selected with a proper control of input coupling conditions, by managing the direction, size, polarization state and shape of the initial pump beam. Because of its nonlinear nature, Kerr-beam self-cleaning strongly affects the spatiotemporal properties of the input pulsed beam [3]. As a result, the brightness, peak power, and degree of polarization can be substantially increased by the self-cleaning process. The main theoretical approach which is able to explain the nonlinear mechanism of beam self-cleaning is based on the periodic self-imaging process, which introduces a periodic modulation in the refractive index of the fiber core, when the Kerr effect comes into play. Such index modulation provides a dynamic long-period grating, which breaks the orthogonality between transverse modes, and phase-matches four-wave mixing processes, which selectively populate a single, low-order fiber mode. Because of the spectral broadening of the initial pump beam, the nonlinear mode coupling process exhibits nonlinear nonreciprocity, and the output power irreversibly remains on a selected transverse mode.

By increasing the input beam power well above the threshold for obtaining KBSC in GRIN MMFs, one observes the generation of a series of spectral sidebands, attributed to a spatiotemporal instability, ranging from the visible to the infrared [4-5]. The periodic evolution of light intensity (or self-imaging) is the mechanism for inducing, via the Kerr effect, a parametric spatiotemporal instability. This instability can be observed irrespective of the dispersion regime of GRIN MMFs, as successfully demonstrated by launching femtosecond or subnanosecond pulses in the anomalous $(1550 \mathrm{~nm})$ or normal $(1064 \mathrm{~nm})$ dispersion regime, respectively [1-5]. In the anomalous dispersion regime, spectral broadening features were explained through the interplay between spatiotemporal multimode soliton oscillations and dispersive wave generation in the GRIN MMF.

On the other hand, tapered optical fibers are well known to provide a number of useful features, including a strong mode confinement capability, small scale diameter and broadband supercontinuum (SC) generation [6,7]. A recent experiment has demonstrated the use of passive GRIN MMF tapers for efficient SC generation [8]. The two major factors leading to mode coupling in long tapered fibers are fiber core radius variation and the lightperturbation-induced refractive index change.

In this work, we report the observation of visible-short infrared SC generation in combination with KBSC in relatively long $(\sim 10 \mathrm{~m})$ tapered $\mathrm{Yb}$-doped MMFs. In our experiment, we used a $9.55 \mathrm{~m}$ long Yb-doped MMF exhibiting a quasi-parabolic core refractive index profile (see fig.1c). The largest input face of the taper was $122 \mu \mathrm{m}$, whereas the smaller one was close to $40 \mu \mathrm{m}$. Our taper was first excited by launching into the largest input face with a $1064 \mathrm{~nm}$ laser source emitting $500 \mathrm{ps}$ pulses with more than $130 \mathrm{~kW}$ of peak power. A CW multimode laser diode (LD) at $940 \mathrm{~nm}$ was also used, in order to pump the rare-earth $\mathrm{Yb}$ ions, which provide gain along the propagation in the tapered fiber. In a first experiment, we observed KBSC in a passive configuration, i.e., the CW pump diode was switched off. When increasing the power of the laser source, the spatial beam pattern at the fiber output evolved significantly, from a speckled beam into a quasi-single mode emission, matching well the fundamental mode profile of the fiber (see fig.1d). Because of the residual absorption of the fiber at $1064 \mathrm{~nm}$, the available output power 
remained limited to $9 \mathrm{~kW}$ (input power: $114 \mathrm{~kW}$ ). In these conditions, no significant frequency conversion was obtained, except for the first Raman Stokes sideband.

(a)

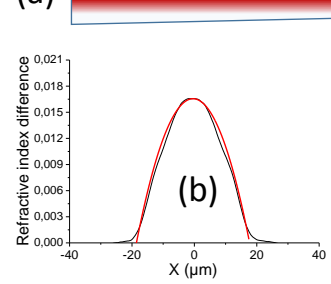

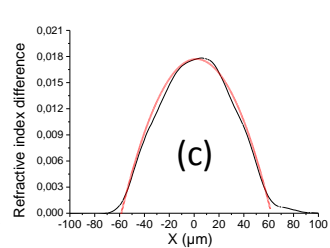
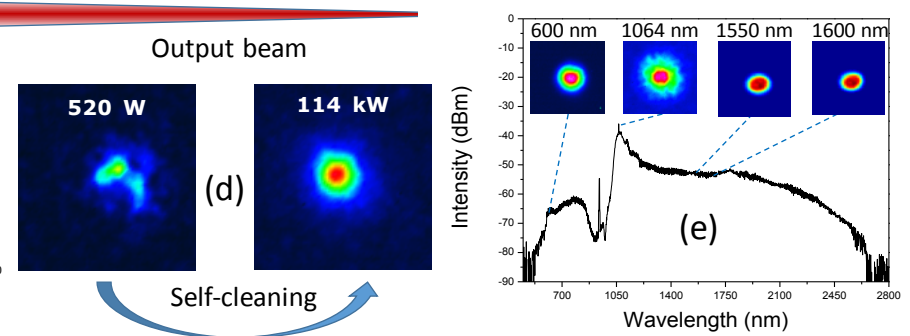

Fig. 1: (a) Doped multimode fiber taper, (b-c) core refractive index profile of small and large diameter, respectively, (d) output beam shape for two input peak powers at $1064 \mathrm{~nm}$ without $\mathrm{CW}$ pump (left: linear propagation, right: nonlinear propagation and spatial self-cleaning) with 9.55 $\mathrm{m}$ long taper, (e) experimental spectra obtained in the active taper with $19.6 \mathrm{~kW}$ input power at $1064 \mathrm{~nm}$ and maximum gain value of $1.3 \mathrm{with}$ $9.55 \mathrm{~m}$ long taper, inset: near-field output beam profile with different bandpass filters.

In a second step, we added the pump at $940 \mathrm{~nm}$ for amplification along the multimode fiber taper. Under this configuration, the input signal wave was amplified mainly over the first meter of propagation. Signal amplification significantly modifies the input beam conditions, and changes via a gain guiding mechanism the energy distribution between modes, thus modifying the self-cleaning dynamics, and distorting the output mode profile. Additionally, by further increasing the CW pump at $940 \mathrm{~nm}$, we also obtained a SC emission between $520 \mathrm{~nm}$ and $2600 \mathrm{~nm}$ (see fig 1 (e)). The visible part of the spectrum is obtained thanks to parametric processes, whereas the infrared spectrum is mainly built-up by a cascade of multimode soliton propagation followed by Raman self-frequency shift.
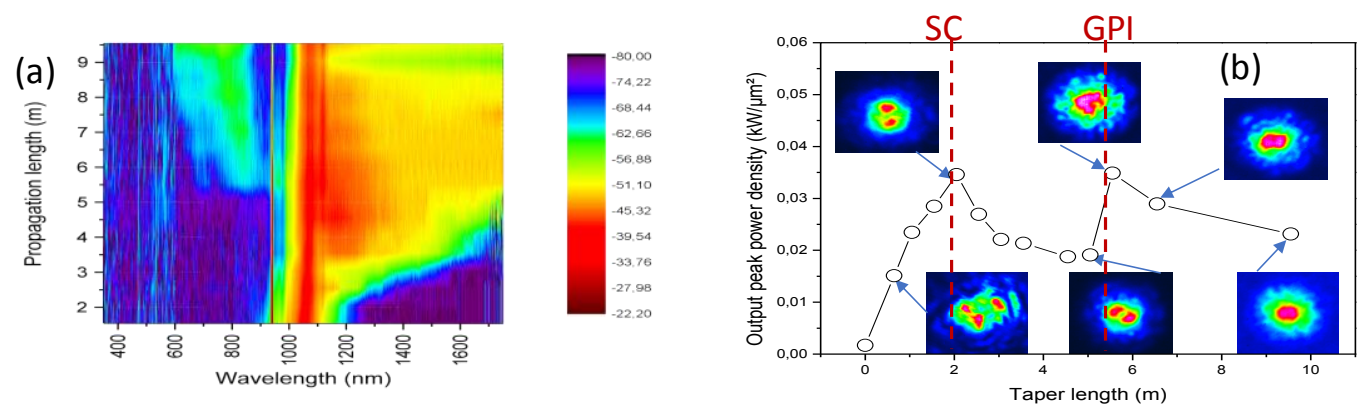

Fig. 2: Experimental results of the cut-back technique with an input peak power of $19.6 \mathrm{~kW}$ in the taper. (a) Experimental spectrum as a function propagation length in the taper, (b) output peak power density at $1064 \mathrm{~nm}$ as function taper length showing beam self-cleaning evolution.

Next, we studied the evolution of beam self-cleaning and SC generation as function of taper length using a cut-back method, and fixing the input peak power at $19.6 \mathrm{~kW}$. Fig. 2.a presents the spectral evolution as a function of the length of the taper. The spectral growth appears from the first meter of fiber (at $2 \mathrm{~m}$ ) with the progressive generation of SC towards the infrared because of the Raman effect, whereas GPI sidebands are generated only after $5 \mathrm{~m}$ of propagation into the taper. Fig2.b shows that the spatial distribution is speckled at the beginning (first meter) of the propagation, then the beam is self-cleaned during its propagation first in a low-order mode $\left(\mathrm{LP}_{11}\right)$, and subsequently in the fundamental $\mathrm{LP}_{01}$ mode.

We acknowledge financial support of: Horiba Medical and BPI france within the Dat@diag project; iXcore research foundation; French National Research Agency ANR Labex ACTION; the European Research Council (grant agreement No. 740355).

\section{References}

[1] L.G. Wright, et al., Nat. Photonics 9, 306 (2015).

[2] K. Krupa, et al., Nat. Photonics 11, 237 (2017).

[3] K. Krupa, et al. Phys. Rev. A 97, 043836 (2018).

[4] G. Lopez-Galmiche, et al., Opt. Lett. 41, 2553 (2016).

[5] K. Krupa et al., Opt. Lett 41, 5785 (2016)

[6] T. Birks, et al., Opt. Lett 25, 1415 (2000).

[7] C. Shi et al., Opt. Express 24, 19473 (2016).

[8] M. A. Eftekhar, et al., in Conference on Lasers and Electro-Optics, Optical Society of America, paper FTh1.M3 (2018) 